\title{
Performance and Applicability of a new Geographic Routing Protocol for Virtual Power Plants
}

\author{
Mouna Rekik \\ Inria Lille - Nord Europe, France \\ CMERP, Sfax, Tunisia. \\ Email: mouna.rekik@inria.fr
}

\author{
Zied Chtourou \\ CMERP, Sfax, Tunisia. \\ Email: ziedchtourou@cmerp.net
}

\author{
Nathalie Mitton \\ Inria Lille - Nord Europe, France. \\ Email: nathalie.mitton@inria.fr
}

\begin{abstract}
The Smart Grid (SG) incorporates communication networks to the conventional electricity system in order to intelligently integrate distributed energy resources (DERs) and allow for demand side management. The move to Smart grid in developing countries has to cope with great disparities of ICT infrastructures even within the same city. Besides, individual DERs are often too small to be allowed access to energy market, likewise power utilities are unable to effectively control and manage small DERs. We propose the use of affordable and scalable wireless communication technology to aggregate geographically sparse DERs into a single virtual power plant. The enrollment of prosumers in the VPP is conditional to financial performance of the plant. Thus, the VPPs are dynamic and are expected to scale up as more and more prosumers are attracted by their financial benefits. the communication network has to follow this progression and therefore to be scalable and rapidly deploy-able. We present a routing algorithm for data communication within the VPP to support centralized, decentralized or fully distributed control of the VPP's DERs.
\end{abstract}

\begin{tabular}{ll}
\multicolumn{1}{c}{ Acronyms and Abbreviations } \\
\hline STEG & Societe Tunisienne d'Electricite et du Gaz \\
SG & Smart Grid \\
ESP & Electricity Service Provider \\
VPP & Virtual Power Plant \\
DR & Demand Response \\
DER & Distributed Energy Ressource \\
DERS & Distributed Enery Ressource and Storage \\
DSO & Distribution System Opertator \\
DG & Distribution Generation \\
ESS & energy storage system \\
MDMS & Meter Data Management System \\
AMI & Advanced Metering Infrastructure \\
ICT & Information and Communication Technology \\
IETF & Internet Engineering Task Force \\
LLN & Low power and Lossy Network \\
ROLL & Routing Over Low power and Lossy networks \\
RPL & Routing Power for Low power and Lossy network \\
DAG & Directed Acyclic Graph \\
GRACO & geographic GReedy routing with ACO based recovery
\end{tabular}

\section{INTRODUCTION}

Conventional electricity grid has been developed based on the assumption that electricity is generated at a few stations generally outside urban areas and transported and distributed to many consumers. It has also been widely accepted that the behavior of energy consumers can be predicted to reasonable extent over hours, days seasons and years.

The Smart Grid (SG) incorporates communication networks to the conventional electricity system in order to intelligently integrate the actions of parties connected to it. Electricity can be generated and consumed anywhere in the grid. The behavior of generators and users is somewhat different from a conventional grid. SG technologies should allow for even better prediction of electricity supply and demand anywhere in the grid. It will provide efficient grid conditions monitoring and control. Moreover, it will provide energy prosumers with provisions to actively interact in managing demand and or production on their side.

In Tunisia, the utility company (STEG) is responsible for the production, transport and distribution of the electricity along with the grid monitoring and maintenance. Its commercial offer is categorized in domestic and industrial profiles. Residential customers have currently access to limited information and almost no incentives or tools to manage their domestic electricity consumption or production. Electricity meters are read manually and each 4 months period, the customer is provided with a bill showing the total balance of energy exchanged with the grid. The utility company practices progressive pricing of electricity for the domestic users. The electricity is charged at a very low price in a limited volume of $\mathrm{kWh}$ (lifeline consumption), and at a much higher price on the $\mathrm{kWh}$ consumed beyond this limit (comfort of consumption). The large business consumer profile is more flexible, it offers variable rates depending on time of use and nature of the activity. Besides, to help balance electricity supply and demand, the utility encourages large power business customers exercise some load-shaving capability, it offers them credits in exchange for curtailing of their electricity consumption on request. These credits are almost equivalent to what would have been the utility's cost for ramping up the electricity production.

In [1], the authors proposed the introduction of a new actor into the Tunisian electric energy market: the Electricity Service Provider (ESP) which is supposed to replace the STEG in tasks related to billing services and renewables integration and promotion. The ESP is not responsible of electricity delivery and grid maintenance. It exploits the utility grid and metering infrastructure within a community as an Internet service provider does with telecom operator. According to this work, the power consumption of consumers within a community will be billed to The ESP by installing a unique industrial meter (IM) just downstream the distribution station serving that community. The ESP measures individual energy transfers through ESP data loggers installed at the customers premises. This concept is conditional to willingness of all customers within the fed segment to adhere to the ESP service. In the present work, we solve this proximity issue by allowing different sparsely localized customers or producers to enroll for the ESP services. We suggest the development of Virtual Power Plant (VPP) run by the ESP. A VPP knits together 
energy resources as if they were a single generator. Any residential energy user or producer can be hooked up. And energy that's generated or not used can also contribute to the VPP's capacity without what these customers have no access to the more convenient business profile. ESP will manage residential consumers and or producers (prosumers) accounts and will provide services such charge curtailment during times of peak demand and storage, energy and capacity trending while drawing from the main grid as needed through enrollment in Demand Response (DR) programs.

In the next section we introduce VPP and Demand Response and the control types. This will allow us to highlight communication needs for the VPP. Then we report a Routing protocol for low power and lossy networks (RPL). This protocol has been standardized by The Internet Engineering Task Force (IETF) and is intended for wide variety of applications including home automation, urban sensor networks and AMI systems[2]. We overview the RPL protocol with the objective of pointing limitations that can hinder its application in the VPP. Then we propose a new routing protocol that can be used for communications for the VPP. Finally, simulation results are presented for different VPP node densities.

\section{Virtual Power Plant}

A VPP is a set of geographically sparse Distributed Energy Resources (DERs) including power generators, controllable loads and storages that are aggregated in a way to perform as a single power facility, as illustrated in figure 1 .

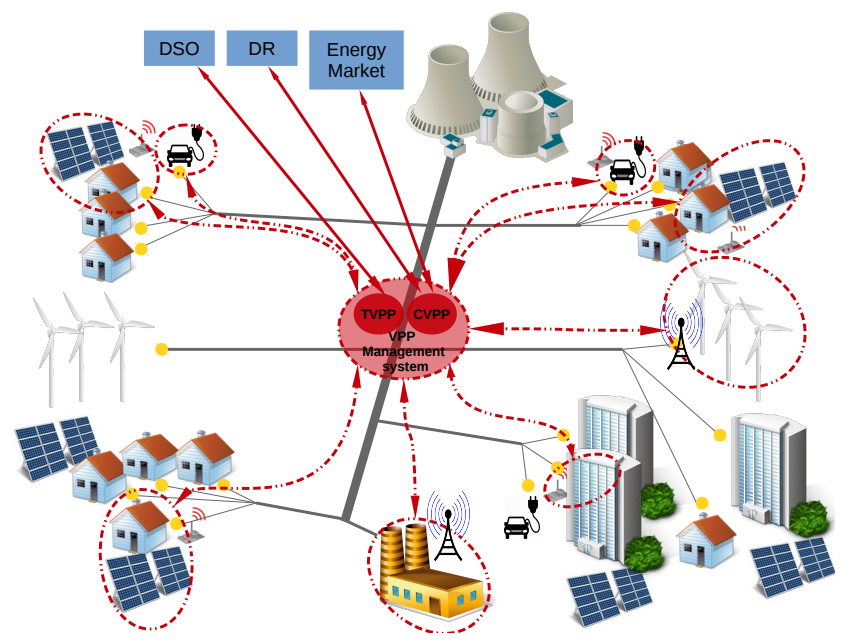

Fig. 1: Illustration of a VPP. The entities of the grid surrounded in red cercles decided to join the VPP

From a technical perspective, the VPP tries to optimize control and coordination, as well as system operation. It interacts directly with the Distribution System Opertator (DSO) providing him with a unique profile of the whole aggregated plant to assist with network management and provide ancillary services. From an economical point of view, VPP describes an economic aggregation of geographically sparse industrial, commercial and residential generation and consumption assets into a single business entity. It integrates Demand Response (DR) programs and demand side management in order to trade the produced energy and or shedded load to the best of the owners interest. Hence, the VPP directly interacts with the energy market.

The development of VPP integrating DERs and DR relies on the implementation of Advanced Metering Infrastructure (AMI), Information and Communication Technologies (ICTs) and Network Automation [3]. The VPP deploys an AMI to allow for bidirectional flow of information between consumers, providers and grid devices part of the VPP such as smart meters and sensor-based controllers of power system equipment. Control of VPPs could be centralized, decentralized and fully distributed. In the centralized control [4] all the knowledge about the DERs production and the energy market is located in the central controller. This gives the VPP a simple way of using DERs to meet grid demand. However, different ownership, and the large number of DERs within a VPP challenge the central controller find optimal control strategy to respond to best prosumers interest.

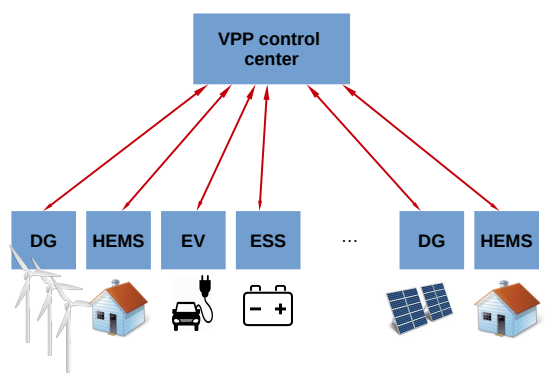

Fig. 2: Centralized controlled VPP

In the distributed control, the complexity is divided vertically within the VPP. [3] introduces a hierarchical model by defining VPPs on different levels. A local VPP supervises and coordinates a limited number of DERs while delegating certain decisions upwards to a higher level VPP. This design requires communication between different neighboring VPPs.

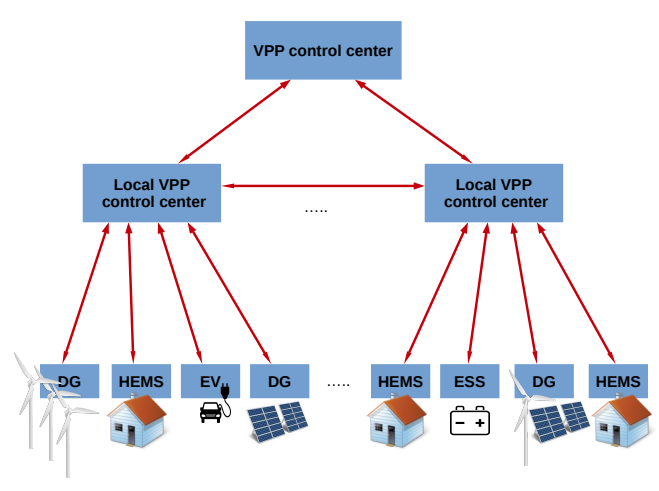

Fig. 3: Distributed controlled VPP: hierarchical model

In the fully distributed control, each DER has communication and processing provisions to participate (and react) independently and smartly in (to) the state of the power system. An example of such control is reported in [5] where minimal coordination between neighboring DERs is reported to be 
enough to reach global optimum state for the whole VPP.

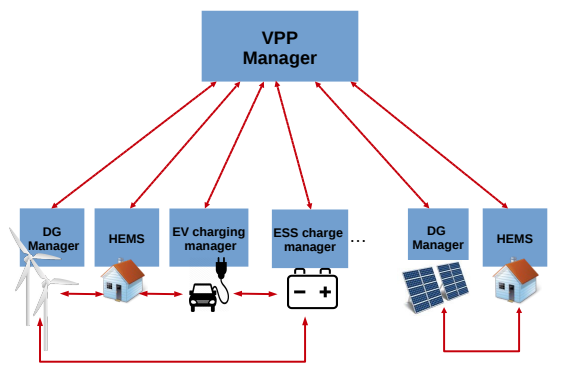

Fig. 4: Fully distributed controlled VPP

The enrollment of prosumers in VPPs relies on the first movers reported satisfaction or desillusion. VPP reported benefits are likely to draw more participants into one VPP. Thus, a VPP could start with a limited number of DERs to rapidly scale to thousands of prosumers. Decentralized control or fully distributed control hold much promise regarding managing a dynamic VPP. Moreover, coordination between DER for number of ancillary services for reactive power management is critical for higher integration of DERs within a powergrid [6]. To support these VPPs and the different associated services, the adopted ICT should be scalable, rapidly deployable and capable of peer to peer communication between DERs to allow for flexible smart distributed control schemes. Wireless communication technology is a good candidate for this AMI network. Indeed, Wireless networks have low installation costs and can rapidly be deployed even over wide areas with data rates and network capacities comparable to wired networks ones. Scalable and energy efficient routing protocols are enablers for these networks in VPP context.

In the next session, we introduce RPL, a routing protocol standardized by The IETF and is intended for wide variety of applications including AMI systems.

\section{Routing Protocol For Low Power AND LOSSY NETWORKS (RPL)}

RPL [7] is a routing protocol for Low power and Lossy Networks (LLNs) designed by the IETF Routing Over Low power and Lossy networks (ROLL) Working Group.

RPL consists of maintaining network state information using one or more Directed Acyclic Graphs (DAGs) [8]. A DAG is a directed graph wherein all edges are oriented in such way that no cycle exists [9]. Each DAG has a root node and client nodes. A rank, calculated using an objective function, is assigned to each client node in the DAG. The rank monotonically increases in the downward direction so the root has the lowest rank. The objective function is defined using various link and node metrics and constraints to compute the best path.

To create a DAG, RPL uses a set of ICMPv6 messages to exchange graph information. The DAG creation starts by the root sending a control packet called DIO (DAG Information Object packet). A DIO message contains information about the DAG such as the DAGID used to identify the DAG as sourced from the DAG root; rank information used by nodes to determine their positions in the DAG relative to each other; objective function identified by an Objective Code Point (OCP) that specifies the metrics used within the DAG and the method for computing DAG rank.

If a client node receives a DIO message and decides to join the DAG, it should add the DIO sender (the previous node traversed by the DIO) to its parent list, compute its own rank (associated with the parent node) Based on information carried in the message (OCP) ant its local state, and broadcast the DIO message with the updated rank information.

In case of loosing connectivity, a node will send out a DAG Information Solicitation (DIS) message and the neighboring nodes that hear it will respond with a DIO message announcing they are a part of a DAG.

Using the constructed DAG, each client node will be able to forward any upward traffic (an upward traffic is a traffic destined to the DAG root) through its parent as the next-hop node.

To create downward routes from the root to a client node, the client node should issue a control message called Destination Advertisement Object (DAO). A DAO packet includes the rank information used by nodes to determine how far away is the destination ( in this case the destinations is the client node). The DAO message will be forwarded to the root using the upward path indicated by the DAG, and all the intermediate nodes record the reverse path information from the DAO message, after that, a complete downward path is established from the root to the client node.

The RPL is optimized for multipoints-to-point and point-tomultipoints communication schemes [10]. For that reason, it was proposed as a routing solution for AMI networks [8][11] where traffic is limited from meters to a concentrator, and from a concentrator to meters. In this context, different implementations (variants) of RPL routing protocol have been proposed in the literature to meet with NAN requirements. The authors of [12] proposed a routing algorithm based on Multiple RPL instances with QoS differentiation at network layer in order to account for NAN's applications requirements. In [13], a modified version of RPL is proposed to support multi-gateway AMI network case. In fact, the standard RPL defines an RPL instance with different disjoint DAGs, each one corresponding a root. For that, the multi-gateway RPL allow a node to join multiple DAGs in order to increase viable routing options [13]. CORPL [9] is another proposed RPL variant for the context of AMI networks, it addresses the problem of instable wireless links and interference effects.

RPL provides only basic support for point-to-point traffic [11]. When a node sends a packet to another node, the packet uses upward route until arriving to a common ancestor at which point it is forwarded in the downward direction to the destination.

To conclude, RPL provides an efficient support of dynamic routing metrics and constraints required by the AMI network. However, establishing and maintaining efficient upward and downward routes according to each possible root can result high overhead specially for large scale networks which may affect the reliability and scalability of the routing solution. Besides, RPL does not provide an optimized point-to-point traffic support. For these reasons, RPL is not suitable for VPP communication case. In the next session, we will propose the use of geographic routing for AMI network in the context of VPP. 


\section{Geographic GREedy Routing With ACO BASED RECOVERY (GRACO)}

\section{A. Geographic routing for VPP communications}

Geographic routing is a class of routing approaches that exploit geographic position in routing decision in stead of topological information. It has been widely used in ad-hoc and wireless sensor networks for its diverse advantages. In fact, GR is a localized routing scheme since packet forwarding decision is achieved by using only information about the position of nodes in the vicinity and the position of the destination node, which minimizes significantly communication overhead. Hence, no additional information has to be stored neither on the nodes on the path, nor in the message which makes GR a memory-less and scalable routing [14]. Besides, GR is also distributed, every node performs the same algorithm [14] As previously discussed, the deployment of a wireless communication network for VPP faces many challenges. First of all, VPP is supposed to support different applications and multiple traffic flows, the communication network should then manage a huge amount of data, thus, the routing solution should deliver the data packets with the minimum overhead possible so it does not affect the network performances. As VPP is a large scale network with highly dynamic topology due to the often expansions to cover larger areas and additional endpoints, it needs a scalable routing solution. Besides, the communication scheme for VPP should be resilient to outdoors harsh communication environment and instability of wireless links. For these reasons, the simplicity, localized and distributed operation and the low overhead, memory-less and scalability features makes GR a very good candidate for VPP.

Some research papers investigated in the application of geographic routing for AMI networks. Perimeter Stateless Routing(GPSR) [15] was proposed in this context. [16] studies of the feasability and the performance of GPSR in NAN scenarios. It was proved that GPSR can support different NAN services in terms of latency and reliability. The authors of [17] compared the performances of GPSR to RPL in the case of NAN network. The simulation results proved that RPL outperforms GPSR in terms of transmission reliability and delay.

\section{B. Overview of GRACO}

GRACO [18] is a geographic routing algorithm that combines a pheromone-assisted greedy forwarding mode and an Ant Colony Optimization (ACO) based recovery mode. In the beginning, GRACO makes the routing decision using geographic greedy forwarding strategy [14]. Greedy forwarding is the simplest implementation of geographic routing. At each step, a node tries to bring the packet closer to the destination, using the euclidean distance as a the progress criteria. Greedy forwarding inherits all the advantages of GR. However it is not always possible, it may lead to a void problem if a packet arrives to a node that has no neighbor closer to destination than itself, the node is, then, stuck and could not progress the packet. GRACO proposes an ACO based strategy to recover from the void problem. The ACO recovery strategy uses two types of ant like packets: Fants to search for a path around the void and Bants to mark the paths found for later use. The Fants will be guided to the direction of the destination using zone concept. Once a Fant arrives to an unstuck node, a node closer to the destination than the stuck node, a Bant is sent to mark the path found by dropping pheromone trails. The recovery is launched with the aim of finding an unstuck node, then, the algorithm switches to greedy forwarding again. The performance of GRACO was compared to the greedy-facegreedy (GFG) routing algorithm. Simulations results presented in [18], shows that GRACO outperforms GFG in term of end-to-end delay, data delivery cost and hop count. Besides, GRACO provides a high data delivery rate.

\section{Simulations And Results}

The performance of GRACO is measured using the WSNET simulator [19].

The simulations are performed within a region downtown the city of Sfax, Tunisia. We choose to incrementally add nodes to the VPP in order to simulate the evolution of the VPP from early stages of a VPP where just a few customers are enrolled in the VPP and the DRs are sparse across the selected region to more advanced stages with denser aggregation of DERs representing a more mature version of the VPP where an important number of prosumers has been attracted by the financial benefits of the DR programs.

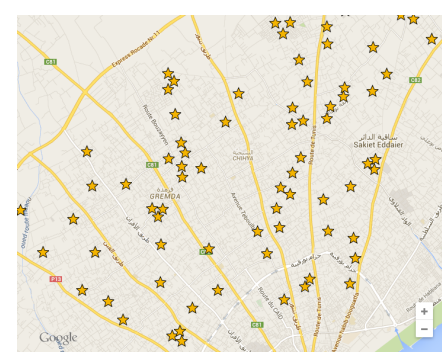

(a)

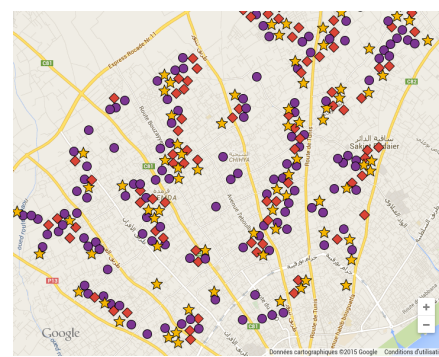

(c)

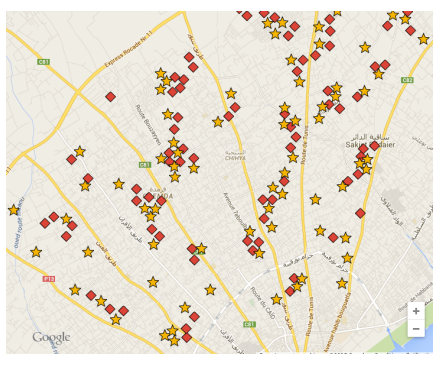

(b)

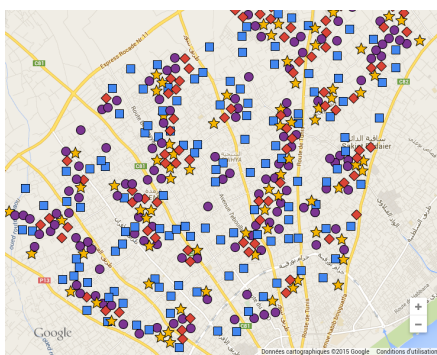

(d)
Fig. 5: Stages of VPP extension within a region downtown the city of Sfax, Tunisia

To vary the nodes degree, we vary their range from $25 \mathrm{~m}$ to $50 \mathrm{~m}$ by steps of $5 \mathrm{~m}$. Each combination of topology and algorithm is run 50 times. Error bars on curves symbolize a $95 \%$ confidence interval. In order to measure the impact of void zones on the algorithm performances, we choose a set of random sources and destinations where there is, necessary, a void to be handled in the routing process. One data packet is sent between a pair of source and destination each 10s.

The performance of the routing protocol is measured in terms of the average route length, the end-to-end delay and the data delivery ratio. The route length is the number of hops a data 


\begin{tabular}{ll} 
Parameter & Value \\
\hline Duration $(\mathrm{s})$ & 300 \\
MAC layer & idealmac and 802.15.4 \\
Interferences & none \\
Density & $10,15,20,25,30,40$
\end{tabular}

TABLE I: Simulation parameters

message needs to go from the source to destination. The endto-end delay is the time interval between a given source sends a packet and the destination receives it. The delivery ratio is the ratio of data packets successfully received by their destinations to all data packets sent by the sources. Table I summarizes the simulation parameters.

The simulation results show that the more the network is dense the better performs the proposed routing protocol.

As the density of the network is increased, shorter paths are found. Specifically, as plotted in figure 6 , the average route length drops from 59 hops at network density 10 down to 11.94 hops at network density 40 .

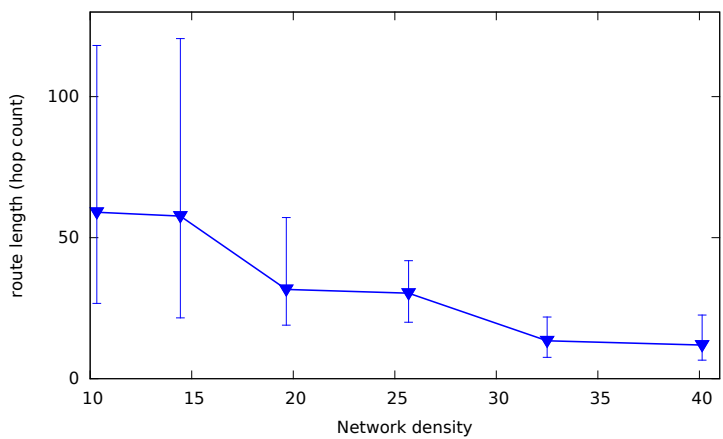

Fig. 6: hop-count

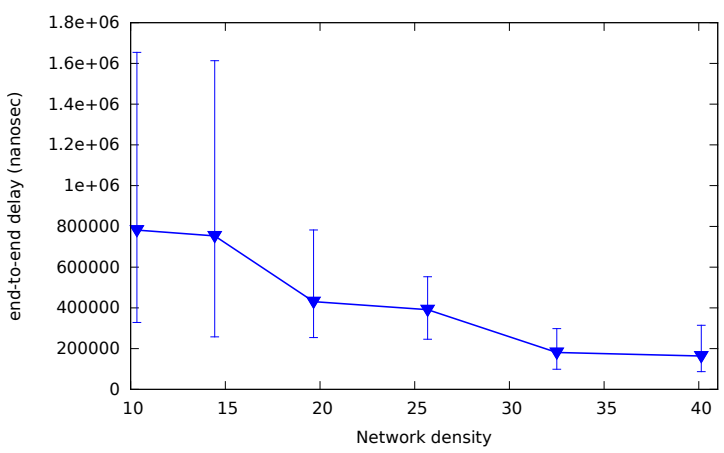

Fig. 7: end-to-end delay

Figure 7 shows the end-to-end delay varing with the network density. The average end-to-end delay reduces from $0.781 \mathrm{~ms}$ at a network density 10 to $0.163 \mathrm{~ms}$ at a network density 40 . The data delivery rate is also improved when increasing network density. In fact, figure 8 shows that GRACO provides a $100 \%$ delivery rate with an ideal mac, however, for 802.15 .4 mac, a high reliability of packet transmission is achieved only in a high density network.

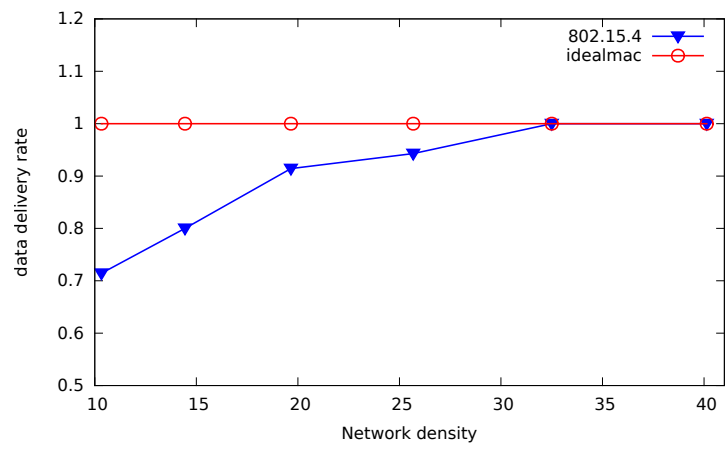

Fig. 8: Data delivery rate

\section{CONCLUSION}

A wireless sensor network is proposed to facilitate installation and development of dynamic virtual power plant. the proposed ACO aided geographic is scalable and ensures point to point communication allowing for versatile control strategies for the VPP such as fully distributed control to overcome the challenges of centralized control. Delivery rate are within the VPP communication requirements. End-to-end delay decreases with increasing network density or VPP size. This is consistent with the need for larger DERs communication traffic within neighborhood for best control strategies decision.

\section{ACKNOWLEDGMENTS}

This work has been partially supported by a grant for the CPER CIA and the MESRST TIC.

\section{REFERENCES}

[1] K. Taouil, T. Jellad, and Z. Chtourou. Communitycoupled microgrids for the implementation of a smart grid in tunisia. international Journal of Energy Technology and Policy, 2014.

[2] D. Popa, J. Jetcheva, N. Dejean, R. Salazar, and J. Hui. Applicability statement for the routing protocol for low power and lossy networks (rpl) in ami networks. Internet draft, 2011.

[3] N. Etherden, M. H. Bollen, and J. Lundkvist. Communication requirements of a virtual power plant using iec 61850 to provide grid services. In Smart Grid Communications (SmartGridComm), IEEE International Conference on, 2013.

[4] D Pudjianto, C Ramsay, and G Strbac. Virtual power plant and system integration of distributed energy resources. Renewable power generation, IET, 1(1):10-16, 2007.

[5] Hongming Yang, Dexin Yi, Junhua Zhao, and Zhaoyang Dong. Distributed optimal dispatch of virtual power plant via limited communication. Power Systems, IEEE Transactions on, 28(3):3511-3512, Aug 2013.

[6] Math $\mathrm{H}$ Bollen and Fainan Hassan. Integration of distributed generation in the power system, volume 80 . John Wiley \& Sons, 2011.

[7] Rpl: Ipv6 routing protocol for low power and lossy networks. http://tools.ietf.org/id/draft-ietf-roll-rpl-19.html, March 2011. 
[8] D. Wang, Z. Tao, J. Zhang, and A.A. Abouzeid. Rpl based routing for advanced metering infrastructure in smart grid. In Communications Workshops (ICC), IEEE International Conference on, pages 1-6, May 2010.

[9] A. Aijaz, H. Su, and A.-H. Aghvami. Corpl: A routing protocol for cognitive radio enabled ami networks. Smart Grid, IEEE Transactions on, 6(1):477-485, Jan 2015.

[10] G. Tuna, V. C. Gungor, and K. Gulez. Wireless sensor networks for smart grid applications: a case study on link reliability and node lifetime evaluations in power distribution systems. International Journal of Distributed Sensor Networks, 2013, 2013.

[11] E. Ancillotti, R. Bruno, and M. Conti. The role of the rpl routing protocol for smart grid communications. Communications Magazine, IEEE, 51(1):75-83, January 2013.

[12] G. Rajalingham, Y. Gao, Q.-D. Ho, and T. Le-Ngoc. Quality of service differentiation for smart grid neighbor area networks through multiple rpl instances. In Proceedings of the 10th ACM Symposium on QoS and Security for Wireless and Mobile Networks, Q2SWinet '14, pages 17-24, New York, NY, USA, 2014. ACM.

[13] P. Thulasiraman. Rpl routing for multigateway ami networks under interference constraints. In Communications (ICC), IEEE International Conference on, pages 44774482, June 2013.

[14] N. Mitton, T. Razafindralambo, and D. Simplot-Ryl. Position-based routing in wireless ad hoc and sensor networks. In Theoretical Aspects of Distributed Computing in Sensor Networks. Springer, 2010.

[15] B. Karp and H.-T. Kung. Gpsr: Greedy perimeter stateless routing for wireless networks. In Proceedings of the 6th annual international conference on Mobile computing and networking, pages 243-254. ACM, 2000.

[16] Q. Ho, G. Rajalingham, and T. Le-Ngoc. Performance and applicability of geographic-based routing in smart grid's neighbor area networks. In Advanced Technologies for Communications (ATC), 2013 International Conference on, pages 215-219. IEEE, 2013.

[17] Q. Ho, Y. Gao, G. Rajalingham, and T. Le-Ngoc. Performance and applicability of candidate routing protocols for smart grid's wireless mesh neighbor area networks. In Communications (ICC), 2014 IEEE International Conference on, pages 3682-3687, June 2014.

[18] M. Rekik, N. Mitton, and Z. Chtourou. Geographic greedy routing with aco recovery strategy graco. Technical report, 2014.

[19] Wsnet simulator. http://wsnet.gforge.inria.fr/. 